\title{
Guía de práctica clínica basada en evidencias para el tratamiento de depresión en adultos en un hospital especializado en salud mental. Lima, Perú \\ Evidence-based clinical practice guideline for the treatment of depression in adults at a specialized mental health hospital. Lima, Peru
}

\author{
Gisella Vargas Cajahuanca,a, Carolina Gallegos Cazorla ${ }^{1, b}$, Carlos Salgado Valenzuela ${ }^{1, c}$, \\ Miriam Salazar de la Cruz ${ }^{1, d}$, Karen Huamán Sánchez ${ }^{1, \mathrm{e}}$, Catherine Bonilla Untiveros ${ }^{1, f}$, \\ Nora Reyes Puma ${ }^{2,3,9}$, Patricia Caballero Ñopo ${ }^{2,3, h}$ \\ ${ }^{1}$ Hospital Víctor Larco Herrera. Lima, Perú. \\ 2 Unidad de Análisis y Generación de Evidencias en Salud Pública, Instituto Nacional de Salud. Lima, Perú. \\ ${ }^{3}$ Facultad de Medicina, Universidad Nacional Mayor de San Marcos. Lima, Perú. \\ a Médico psiquiatra, especialista en psiquiatría de niños y adolescentes \\ ${ }^{\mathrm{b}}$ Médico psiquiatra \\ ${ }^{\circ}$ Médico psiquiatra, magister en gerencia social \\ ${ }^{d}$ Médico familiar y comunitario, maestra en gestión estratégica de la calidad y auditoria médica \\ ${ }^{e}$ Licenciada en Enfermería \\ ' Licenciada en nutrición, master in science \\ ${ }^{9}$ Médico, magister en salud pública \\ ${ }^{\mathrm{h}}$ Médico infectólogo, master in science
}

An Fac med. 2019; 80(1):123-30. / DOI: https://doi.org/10.15381/anales.v80i1.15882

\author{
Correspondencia: \\ Karen Huamán Sánchez \\ Instituto Nacional de Salud, Unidad de \\ Análisis y Generación de Evidencias \\ en Salud Pública (UNAGESP) \\ Av. Defensores del Morro 2268 (Ex \\ Huaylas) - Chorrillos, Lima. \\ Tel. (511) 7480000 Anexo 2207 \\ khuamans@ins.gob.pe
}

Recibido: 15 de febrero 2019

Aprobado: 28 de febrero 2019

Publicación en línea: 27 de marzo 2019

Conflictos de interés: Los autores declaran no tener conflictos de interés.

Fuente de financiamiento: Instituto Nacional de Salud, Ministerio de Salud del Perú. Hospital Victor Larco Herrera, Ministerio de Salud del Perú.

Contribuciones de autoría: Todos los autores participaron en el proceso de elaboración de la guía. Karen Huamán redactó la primera versión del artículo. Todos los autores participaron en la discusión de los estudios y la formulación de las recomendaciones. Todos los autores revisaron y aprobaron la versión final del artículo.

Citar como: Vargas G, Gallegos C, Salgado C, Salazar M, Huamán K, Bonilla C, Reyes N, Caballero P. Guía de práctica clínica basada en evidencias para el tratamiento de depresión en adultos en un hospital especializado en salud mental. Lima, Perú. An Fac med. 2019;80(1):123-30. DOl: https:/doi. org/10.15381/anales.v80i1.15882

\section{Resumen}

Introducción. La depresión es un trastorno mental que en su forma más grave puede terminar en el suicidio. Objetivo. La presente guía de práctica clínica basada en evidencias (GPC-BE) brinda recomendaciones para el tratamiento farmacológico y psicoterapéutico de la depresión en adultos. Métodos. Se elaboró una GPC-BE adaptada mediante un proceso sistemático, riguroso y transparente con un grupo elaborador conformado por metodólogos y psiquiatras expertos en el manejo de la enfermedad. La guia cuenta con siete preguntas clínicas y 10 recomendaciones; se realizó la búsqueda y preselección de GPC que respondan al alcance y objetivos planteados, las mismas que fueron evaluadas mediante el instrumento "Appraisal of Guidelines for Research and Evaluation II" (AGREE-II), seleccionándose la guía de mejor calidad metodológica para su adaptación. La búsqueda sistemática de evidencias para responder las preguntas clinicas se realizó en múltiples bases de datos: MEDLINE/PubMed, EMBASE/Ovid, Cochrane Library, LILACS y SciELO. Las evidencias fueron seleccionadas y analizadas críticamente por pares clínicos y metodológicos, las recomendaciones fueron elaboradas mediante la metodologia Grading of Recommendations Assessment, Development, and Evaluation (GRADE). Resultados. Con la participación de un panel de expertos clínicos de diferentes hospitales de referencia en el manejo de depresión, se elaboraron 10 recomendaciones dirigidas al inicio del tratamiento farmacológico y psicoterapéutico, la terapia combinada, psicoterapia individual-grupal, y eventos adversos de la farmacoterapia en los pacientes adultos con depresión. Conclusiones. El presente artículo es un resumen de la guia de práctica clínica basada en evidencias para el tratamiento de depresión en adultos para un hospital público peruano especializado en salud mental.

Palabras clave: Depresión; Salud Mental, Guía de Práctica Clínica; Medicina Basada en la Evidencia

\section{Abstract}

Introduction. Depression is a mental disorder that in its most severe form can end in suicide. Objective. This Evidence-based Clinical Practice Guideline (EB-CPG) provides recommendations for the pharmacological and psychotherapeutic treatment of depression in adults. Methods. An adapted EB-CPG was elaborated through a systematic, rigorous and transparent process with a team of methodologists and psychiatrists experts in clinical management of the disease. The guide has seven clinical questions and 10 recommendations, the search and preselection of CPGs was carried out responding to the scope and objectives, which were evaluated through the "Appraisal of Guidelines for Research and Evaluation II" (AGREEII), and the guide with the best methodological quality was selected for methodological adaptation. A systematic search for evidence to answer the clinical questions was made in multiple databases: MEDLINE / PubMed, EMBASE / Ovid, Cochrane Library, LILACS and SciELO. The evidences were selected and critically analyzed by clinical and methodological pairs, the recommendations were elaborated through the Grading of Recommendations Assessment, Development, and Evaluation (GRADE) methodology. Results. With the participation of a panel of clinical experts from different reference hospitals in the clinical management of depression, 10 recommendations about the beginning of pharmacological and psychotherapeutic treatment, combined therapy, individual-group psychotherapy, and adverse drug-therapy events were approved for adult patients with depression. Conclusions. This article is a summary of the evidence-based clinical practice guidelines for the treatment of depression in adults in a Peruvian mental health public hospital.

Keywords: Depression; Mental Health; Practice Guideline; Evidence-Based Medicine 


\section{INTRODUCCIÓN}

La depresión es una enfermedad que afecta a más de 300 millones de personas en el mundo'; muchas veces los pacientes con esta enfermedad son estigmatizados, impidiéndoles acceder a una atención oportuna y adecuada ${ }^{2}$. En el Perú, aproximadamente más del 90\% de pacientes con depresión no recibe un tratamiento eficaz y adecuado, debido a causas como las limitaciones en el diagnóstico pudiendo llegar al sobretratamiento. Otro problema paralelo, es el escaso número de médicos especialistas en psiquiatría a nivel nacional, pues la mayoría se ha concentrado en grandes ciudades del país ${ }^{1,2,3}$.

La depresión en adultos es causa frecuente de consulta médica ambulatoria, ocupando uno de los primeros lugares como tema de salud para el desarrollo de guías de práctica clínica, durante el proceso de priorización llevado a cabo en el Hospital Víctor Larco Herrera (HVLH). En este proceso también participaron profesionales psiquiatras y psicólogos de otras instituciones del Ministerio de Salud (MINSA) y del Seguro Social de Salud (ESSALUD).

En nuestro país, la última guía nacional sobre depresión fue aprobada por el MINSA en el año 2008, conteniendo recomendaciones orientadas solo al tratamiento farmacológico ${ }^{4}$. Sin embargo, en la práctica clínica actual, los hospitales vienen aplicando diferentes tipos de psicoterapia además del uso de medicamentos antidepresivos, basándose en evidencias de guías internacionales. Por otro lado, la reciente creación de los "Centros de Salud Mental Comunitarios" del MINSA, que cuentan con profesionales psiquiatras y equipo multidisciplinario, constituyen una oportunidad para la protección y tratamiento de pacientes con trastornos mentales y la promoción de la salud mental en el primer nivel de atención ${ }^{5,6}$.

En este contexto, las autoridades y profesionales del Hospital Víctor Larco Herrera, institución especializada en salud mental, en colaboración con el Instituto Nacional de Salud, a través de la Unidad de Análisis y Generación de Evidencias en Salud Pública (UNAGESP), elaboraron la "Guía de práctica clínica basada en evidencias (GPC-BE) para el tratamiento de la depresión en adultos", que fue aprobada mediante Resolución Directoral del hospital N¹32-2018-DG$\mathrm{HVLH}^{7}$. Esta guía contiene recomendaciones que serán aplicadas por el personal clínico asistencial del hospital y los centros comunitarios de salud mental.

\section{METODOLOGÍA}

La presente GPC-BE se realizó mediante un proceso sistemático, riguroso y transparente aplicando la metodología GRADE según las pautas del Ministerio de salud del Perú ${ }^{8}$ como se detalla en su versión extensa disponible en: https://www. larcoherrera.gob.pe/images/documentos/resoluciones/2018/RD_132_2018_ DG_HVLH.pdf

Conformación de los grupos y panel de expertos

Para el desarrollo de la guía se conformaron dos grupos y un panel de expertos. El primero fue el grupo metodológico (GM) integrado por profesionales del Instituto Nacional de Salud que tuvieron a cargo el proceso de transferencia tecnológica y asesoramiento técnico en todo el proceso. El segundo fue el grupo elaborador (GE) integrado por profesionales psiquiatras del hospital expertos en el manejo clínico de la enfermedad y responsables directo de la elaboración de la guía. El panel de expertos fue un tercer grupo de profesionales expertos clínicos conformado por médicos psiquiatras, médicos de familia, psicólogos, psicoterapeutas, trabajadoras sociales, representantes de la Dirección Nacional de Salud Mental del Ministerio de Salud, Instituto Nacional de Salud Mental Honorio Delgado-Hideyo Noguchi, Hospital Hermilio Valdizan y centros de salud comunitarios, quienes fueron convocados para dar opinión técnica durante el proceso.

La presente guía tuvo el objetivo primordial de brindar recomendaciones basadas en evidencia para actualizar el enfoque integral del tratamiento antidepresivo incluyendo tanto el tratamiento farmacológico como psicoterapéutico.

Asimismo, el alcance de la guía es el tratamiento, porque existe consenso en aplicar las directrices diagnosticas (Figura 1) que se encuentran definidas por la Clasificación Internacional de Enfermedades de la Organización Mundial de la Salud - décima versión (CIE-10) como referencia para Perú ${ }^{9,10}$.

\section{Formulación de preguntas PICO y evaluación de guías internacionales}

Las preguntas clínicas se formularon en formato PICO (population, intervention, comparison y outcome, por sus siglas en inglés), fueron validadas con la participación de actores clave expertos en el tratamiento de adultos con depresión y de tomadores de decisión del Ministerio de Salud. Estas preguntas fueron priorizadas según el alcance terapéutico de la GPC.

Se realizó una búsqueda sistemática de guías de práctica clínica en bases de datos, metabuscadores y repositorios de GPC en páginas web de instituciones desarrolladoras de guías reconocidas a nivel internacional ${ }^{11}$, identificándose un total de 31 guías internacionales y una nacional. Primero se realizó una selección basada en el título y resumen de las guías, aplicando metodología por pares del GM del INS y el GE del HVLH, eliminándose 28 guias, por no cumplir con los objetivos a desarrollar; con las guias preseleccionadas se realizó la evaluación de calidad utilizando el instrumento AGREE $\|^{12}$ a través de su plataforma virtual (http://www. agreetrust.org). Así, se decidió realizar un proceso de adaptación para la presente guía, aplicando la metodología por pares el GM y el GE. Del total de tres guias seleccionadas, dos tuvieron calificación de AGREE del 60\% o más, lo que las califica como guías aprobadas para adaptación. Estas son las guias de "Detección temprana y diagnóstico del episodio depresivo y trastorno depresivo recurrente en adultos. Atención integral de los adultos con diagnóstico de episodio depresivo o trastorno depresivo recurrente"13 de Colombia y "GPC sobre el Manejo de la Depresión en el Adulto" ${ }^{14}$ de España. Concluida la fase metodológica se decidió aplicar la metodología de adaptación.

\section{Proceso de revisión y síntesis del conjunto de la evidencia}

La búsqueda sistematica se realizó en múltiples bases de datos como: MEDLINE/ PubMed, EMBASE/Ovid, Cochrane Library, 


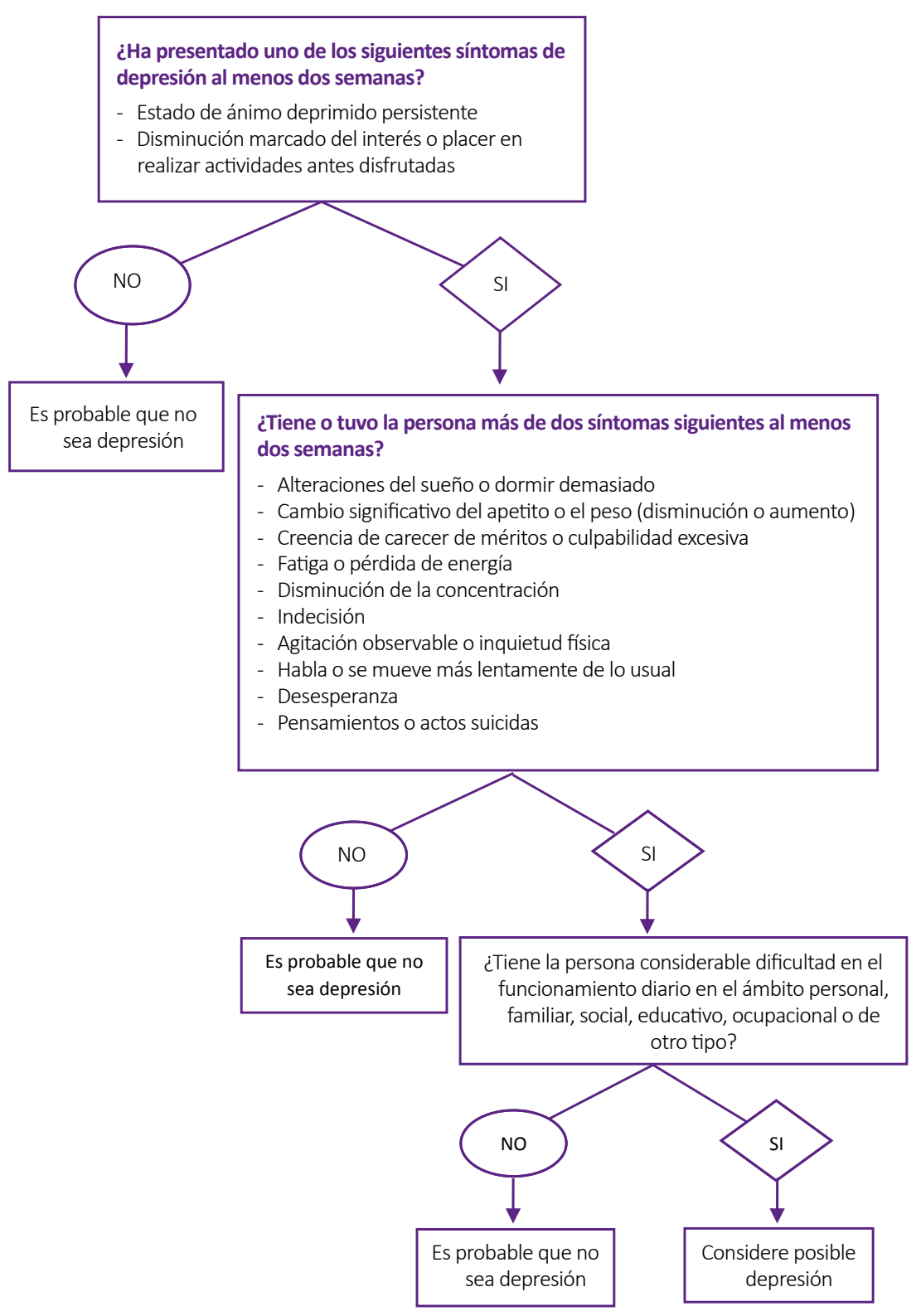

Figura 1. Diagnóstico de la depresión según CIE-10

Adaptado de Organización Panamericana de la Salud, Organización Mundial de la Salud, Mental Health Gap Action Programme. Guía de intervención mhGAP para los trastornos mentales, neurológicos y por consumo de sustancias en el nivel de atención de salud no especializada.Versión 2.0. 2017

LILACS y SciELO, tomando en consideración los niveles de jerarquía de evidencia, iniciando con revisiones sistemáticas, metaanálisis, ensayos clínicos aleatorizados y estudios de observacionales.

\section{Gradación del cuerpo de la evidencia}

El conjunto de evidencia resultante de la búsqueda fue seleccionada por título y resumen por dos miembros del GEG del HVLH y dos metodólogos del INS, luego se
Development, and Evaluation (GRADE), que permitió integrar la mejor evidencia y la experiencia clínica de los miembros del GE. Para cada pregunta se elaboraron tablas de resumen de hallazgos y evidencia GRADE (Summary of Findings tables/ SoF) y marcos GRADE de la evidencia a la decisión (Evidence to Decision/ EtD) a través de la plataforma GRADEpro (https://gradepro.org/), cuya interpretación de niveles de evidencia y fuerza de recomendación se muestran en las tablas 1 y 2 . Este proceso de formulación de recomendaciones fue enriquecido con la aplicación de la metodología del diálogo deliberativo, en los cuales participó el panel de expertos clínicos en conjunto con el grupo metodológico y el grupo elaborador de la guía ${ }^{15,16,17}$.

\section{Incorporación de la perspectiva de} los pacientes

El GE decidió utilizar una guía de entrevista semi-estructurada validada por clínicos y metodólogos con preguntas abiertas dirigidas a recolectar la percepción de los pacientes en relación a la elección del tipo de tratamiento y los eventos adversos de los mismos. Fue aplicada de manera voluntaria, intencional por oportunidad a dos pacientes con tratamiento controlado y en seguimiento, en consulta externa de psiquiatría del HVLH. Adicionalmente se realizó una búsqueda de evidencia cualitativa sobre las intervenciones.

\section{RECOMENDACIONES}

La presente guía cuenta con 7 preguntas PICO y 10 recomendaciones orientadas al tratamiento farmacológico y psicoterapéutico de adultos con depresión, las mismas que se muestran en las tablas 3 y 4.

La metodología GRADE utilizada permite integrar la evidencia científica, la experiencia clínica y la perspectiva de los pacientes en el desarrollo de recomendaciones en las guias de práctica clínica. A continuación se describe el proceso de desarrollo: 
Tabla 1. Grading of Recommendations Assessment, Development, and Evaluation (GRADE): interpretación de niveles de evidencia

Nivel de evidencia GRADE

Alta

\begin{tabular}{ll}
\hline Moderada & $\begin{array}{l}\text { Probablemente puedan existir nuevos estudios que influyan en la } \\
\text { confianza de los resultados estimados y puedan modificarlo. }\end{array}$ \\
\hline Baja & $\begin{array}{l}\text { Es muy probable que nuevos estudios influyan en la confianza de } \\
\text { los resultados estimados y los modifiquen. }\end{array}$ \\
\hline Muy baja & Cualquier resultado estimado es muy incierto.
\end{tabular}

\section{INICIO DE TRATAMIENTO}

Pregunta 1. ¿Cuándo está indicado iniciar un tratamiento con antidepresivos en pacientes con diagnóstico de depresión?

De la evidencia científica a la práctica clínica

Una revisión sistematica con metaanálisis de 6 estudios reportó resultados significativos sobre el beneficio del uso de medicamentos antidepresivos en pacientes con depresión muy severa, el tamaño del efecto de la intervención tuvo variación en función de la severidad de los síntomas. Todos los estudios utilizaron escala de Hamilton (HDRS) para medir la severidad de los síntomas. Este metaanálisis sustentó el uso de antidepresivos cuando las depresiones son muy severas. Por otro lado, los pacientes con síntomas leves o moderados no evidenciaron cambios significativos con la intervención, la evidencia fue de baja calidad ${ }^{18}$.

Beneficios clínicos y daños potenciales de esta recomendación

En los pacientes con depresión leve, los efectos adversos son más fácilmen- te percibidos ya que el beneficio no es evidente, en cambio, en pacientes con depresión muy severa los beneficios son más claros y los eventos adversos son mejor tolerados. El panel de expertos de la guía sugirió que en los casos de depresión moderada sería necesario considerar la presencia de comorbilidades para decidir la intervención.

Pregunta 2. ¿Cuándo está indicado iniciar un tratamiento psicoterapéutico en pacientes con diagnóstico de depresión?

De la evidencia científica a la práctica clínica

Una revisión sistematica con metaanálisis reportó que el tratamiento psicoterapéutico fue consistentemente superior a condiciones control en el manejo del trastorno depresivo. El tratamiento psicológico mostró ser superior a las condiciones control que fueron: lista de espera, manejo usual u otras intervenciones. El tamaño del efecto fue mayor a medida que la severidad del cuadro depresivo era mayor antes del tratamiento (evaluado por criterios del manual diag-
Tabla 2. Grading of Recommendations Assessment, Development, and Evaluation (GRADE): interpretación de fuerza de recomendaciones

\begin{tabular}{ll} 
Fuerza de la recomendación & \multicolumn{1}{c}{ Significado } \\
Fuerte a favor & $\begin{array}{l}\text { Los efectos deseables superan los efectos indeseables. } \\
\text { Se recomienda hacerlo }\end{array}$ \\
\hline Condicional a favor & $\begin{array}{l}\text { Los efectos deseables probablemente superan los efectos } \\
\text { indeseables. } \\
\text { Se sugiere hacerlo }\end{array}$ \\
\hline Condicional en contra & $\begin{array}{l}\text { Los efectos indeseables probablemente superan los efectos } \\
\text { deseables. } \\
\text { Fuerte en contra }\end{array}$ \\
\hline
\end{tabular}

nóstico y estadístico de los trastornos mentales IV/DSM IV o por punto de corte en las escalas Beck Depression Inventory/ BDI o Hamilton Depression Rating Scale/ HDRS). En el caso de la activación comportamental y la terapia interpersonal, la evidencia fue de baja calidad ${ }^{19}$. Otra revisión sistematica con metaanálisis reportó que al evaluar la eficacia de la terapia cognitiva conductual, no se incrementan los resultados conforme a la severidad basal de la depresión; cuando se midió tanto por reporte de pacientes o escalas clínicas, la evidencia fue de alta calidad ${ }^{20}$.

Beneficios clínicos y daños potenciales de esta recomendación

Los efectos beneficiosos esperados fueron considerados moderados, mientras que los efectos indeseables fueron pequeños. En el caso de la depresión severa se consideró que podría ser riesgoso intervenir sólo con psicoterapia. La intervención psicoterapéutica sola como inicio de tratamiento es beneficiosa en la depresión leve y en la moderada, pero es riesgosa en la depresión severa ya que puede existir riesgo latente de ideación suicida.

\section{TERAPIA COMBINADA}

Pregunta 3. En pacientes adultos con diagnóstico de depresión moderada o grave, ¿el tratamiento psicoterapéutico combinado con tratamiento farmacológico (terapia combinada), comparado con farmacoterapia sola, es más efectivo en la remisión de síntomas, mejor calidad de vida, recuperación funcional, disminución del riesgo suicida, intento de suicidio y suicidios?

De la evidencia científica a la práctica clínica

Una revisión sistemática con metaanálisis que comparaba las tasas de deserción de los tratamientos farmacológicos sólos o combinados en 19 estudios, reportó tasas de abandono significativamente menor en el tratamiento combinado en comparación con la farmacoterapia sola (OR=0,65; IC 95\% 0,50-0,83), casi $\sin$ heterogeneidad $(Q=18,11, N S$, $\left.\mathrm{I}^{2}=0,62\right)^{21}$. La calidad de la evidencia fue moderada, asimismo, las intensidades y 
Tabla 3. Lista de Preguntas PICO de la guia de práctica clínica basada en evidencias para el tratamiento de depresión en adultos.

\begin{tabular}{lll} 
Tema & \multicolumn{1}{c}{$\mathbf{N}^{\circ}$} & \multicolumn{1}{c}{ Pregunta } \\
Inicio de & Pico 1 & $\begin{array}{l}\text { ¿Cuándo está indicado iniciar un tratamiento con antidepresivos en } \\
\text { pacientes con diagnóstico de depresión? }\end{array}$ \\
\cline { 2 - 3 } & Pico 2 & $\begin{array}{l}\text { ¿Cuándo está indicado iniciar un tratamiento psicoterapéutico en } \\
\text { pacientes con diagnóstico de depresión? }\end{array}$ \\
\hline \multirow{2}{*}{$\begin{array}{l}\text { Terapia } \\
\text { combinada }\end{array}$} & $\begin{array}{l}\text { En pacientes adultos con diagnóstico de depresión moderada o } \\
\text { grave, ¿̇el tratamiento psicoterapéutico combinado con tratamiento } \\
\text { farmacológico (terapia combinada), comparado con farmacoterapia } \\
\text { sola, es más efectivo en la remisión de síntomas, mejor calidad de } \\
\text { vida, recuperación funcional, disminución del riesgo suicida, intento } \\
\text { de suicidio y suicidios? }\end{array}$ \\
\hline
\end{tabular}

En pacientes adultos con diagnóstico de depresión moderada o grave, ¿̇cuál es el tratamiento psicoterapéutico solo o en combinación con farmacoterapia, más efectivo considerando como opciones

Pico 4 terapia cognitivo conductual, terapia interpersonal o terapia racional emotiva; para lograr remisión de síntomas, mejor la calidad de vida, recuperación funcional, disminución del riesgo suicida, intento de suicidio y suicidio?

Psicoterapia individual y grupal

En pacientes adultos con diagnóstico de depresión moderada o grave ¿Los grupos de ayuda mutua son más efectivos en comparación con

Pico 5 la terapia familiar sistémica para lograr remisión de síntomas, mejor la calidad de vida, recuperación funcional, disminución del riesgo suicida, intento de suicidio y suicidios?

¿Cuánto es el tiempo adecuado de tratamiento con psicoterapia (o

Pico 6 número de sesiones necesarias) para obtener respuesta terapéutica, remisión de síntomas y recuperación funcional en pacientes con diagnóstico de depresión?

Efectos

adversos de la

farmacoterapia
En pacientes adultos con depresión leve o moderada, ¿El uso

Pico 7 de farmacoterapia es seguro en términos de interacciones medicamentosas y reacciones adversas? duraciones de los tratamientos fueron variables del mismo modo que los tiempos de seguimiento de los pacientes.

\section{Beneficios clínicos y daños potencia-} les de esta recomendación

La terapia combinada presenta beneficios para el usuario, ya que interviene en el componente biológico del trastorno, así como el componente psicológico, brindando un tratamiento integral. El tratamiento farmacológico, aun no teniendo efectos inmediatos, brinda una mejoría de los síntomas aproximadamente a las dos semanas. Mientras que la psicoterapia, puede tardar un mayor tiempo para evidenciar respuesta22. Los daños potenciales de la terapia combinada son la presentación de eventos adversos de la medicación, como disminución de la libido, efectos gastrointestinales o una respuesta parcial de la sintomatología. Respecto a la psicoterapia, el daño potencial, no es propio del procedimiento, si no de quien la aplica o del sistema que la administra. Es decir, ausencia de personal adecuadamente entrenado.

\section{PSICOTERAPIA INDIVIDUAL Y GRUPAL}

Pregunta 4. En pacientes adultos con diagnóstico de depresión moderada o grave, ¿cuál es el tratamiento psicoterapéutico solo o en combinación con farmacoterapia, más efectivo considerando como opciones terapia cognitivo conductual, terapia interpersonal o terapia racional emotiva; para lograr remisión de síntomas, mejor la calidad de vida, recuperación funcional, disminución del riesgo suicida, intento de suicidio y suicidio?

\section{De la evidencia científica a la práctica clínica}

Se seleccionó una revisión sistemática con metaanálisis con el objetivo de determinar la efectividad de la terapia cog- nitiva conductual (TCC) en comparación con otras psicoterapias y farmacoterapia con antidepresivos; aunque el metaanálisis no incluyó la terapia racional emotiva (TRE), sí tenía información sobre la terapia interpersonal (TIP). Los resultados reportaron que la única psicoterapia con efectos similares a la TCC fue la terapia psicodinámica. Por otro lado, al comparar TCC con TIP, los estudios mostraron que el efecto de la TIP no fue significativamente superior a la TCC. Al evaluar los efectos de las intervenciones psicoterapéuticas con el inventario de depresión de Beck, los estudios reportaron que en la comparación de TCC con TIP, los efectos más grandes se presentaron en las terapias de soporte y la terapia psicodinámica mientras que con la TIP los efectos no fueron significativos23.

En relación a la terapia racional emotiva, un ensayo clínico reportó que luego de las 14 semanas de tratamiento el 50\% (28/56) de pacientes del grupo de la TCC presentó remisión de síntomas en comparación con el 43,9\% (25/27) de pacientes de este grupo de TRE. A los seis meses de seguimiento de las intervenciones, los efectos no variaron significativamente para la TCC, es así que el 52,1\% (25/48) de pacientes del grupo de la TCC presentó remisión de síntomas; sin embargo, en el grupo de pacientes con TRE existió un incremento de la remisión de síntomas en el 49\% (24/49) de pacientes. Los desenlaces fueron medidos con ambos instrumentos mencionados anteriormente24. El estudio fue de calidad moderada ya que no existió aleatorización en la selección de los pacientes.

Beneficios clínicos y daños potenciales de esta recomendación

Tanto los beneficios y los daños potenciales son considerados pequeños. Es importante señalar que existen estudios que demuestran que más que el tipo de psicoterapia empleada es la relación paciente - terapeuta lo que incrementa la efectividad de la psicoterapia siempre y cuando esta sea brindada adecuadamente por un personal capacitado.

Pregunta 5. En pacientes adultos con diagnóstico de depresión moderada o grave ¿Los grupos de ayuda mutua son más efectivos en comparación con la 
Tabla 4. Recomendaciones de la guia de práctica clínica basada en evidencias para el tratamiento de depresión en adultos.

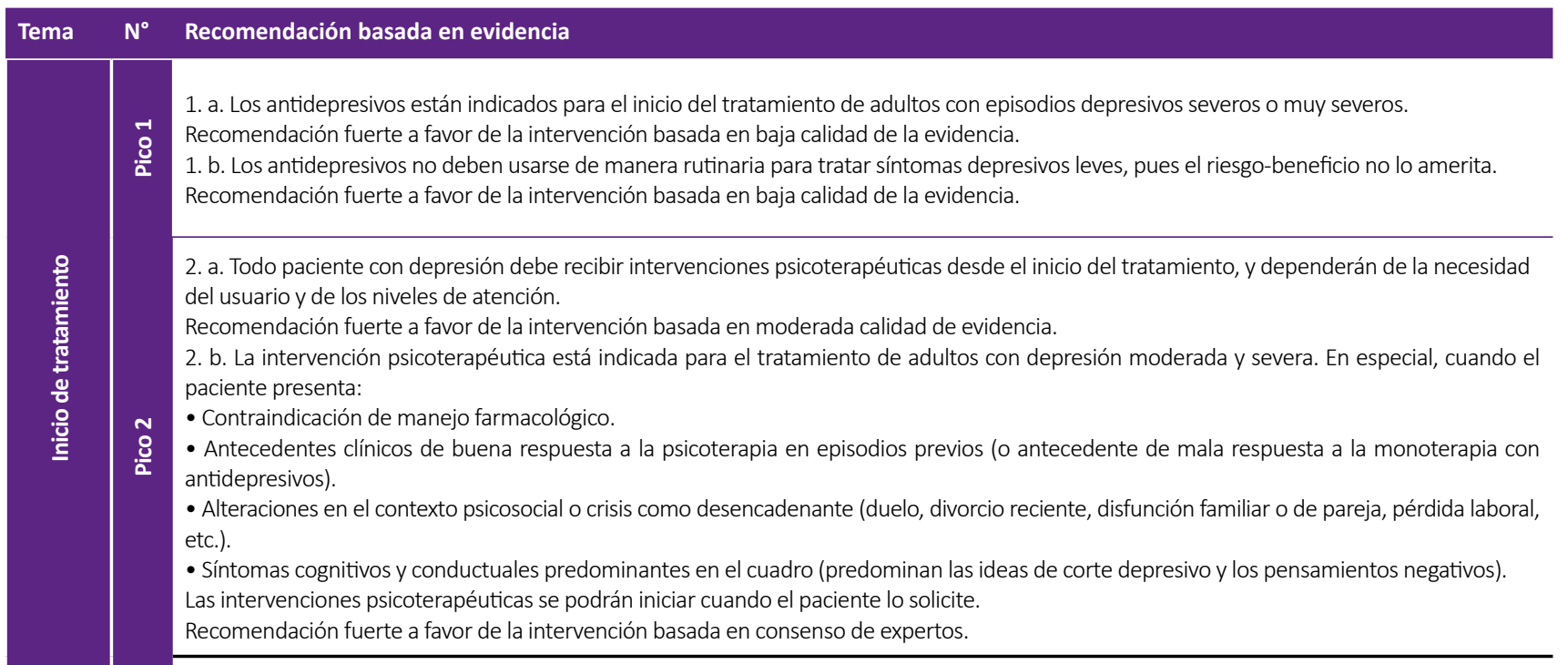

3. a. En pacientes adultos con depresión severa, se recomienda usar la terapia combinada (terapia farmacológica y psicoterapia).

m Recomendación fuerte a favor de la intervención basada en moderada calidad de la evidencia.

¿ 3. b. En pacientes adultos con depresión moderada es necesario considerar la pertinencia de brindar una terapia combinada (terapia farmacológica y psicoterapia).

Recomendación fuerte a favor de la intervención basada en moderada calidad de la evidencia.

4. El panel de la guía recomienda terapia cognitiva conductual, terapia interpersonal o terapia racional emotiva en pacientes adultos con depre-

sión moderada o severa, según el criterio clínico y las necesidades del paciente.

Recomendación condicional a favor de la intervención o la comparacion basada en moderada calidad de la evidencia.

n 5. El panel de la guía sugiere implementar grupos de ayuda mutua como una intervención psicosocial en pacientes adultos con diagnóstico de 8 depresión moderada psicosocial.

Recomendación condicional a favor de la intervención basada en muy baja calidad de evidencia.

6. La duración de las intervenciones psicoterapéuticas debe ser entre 16-20 sesiones en 3-4 meses. El número de sesiones puede ampliarse dependiendo de las necesidades del paciente.

Recomendación fuerte a favor de la intervención basada en moderada calidad de la evidencia.

7. Se recomienda iniciar el tratamiento de la depresión severa en adultos con medicamentos Inhibidores selectivos de la recaptación de serotonina (IRSS).

Recomendación fuerte a favor de la intervención basada en alta calidad de la evidencia.

Consideraciones:

Al prescribir un antidepresivo para el tratamiento de la depresión severa en adultos, el médico debe decidir el tipo de fármaco según el perfil de efectos adversos y el potencial de interacciones medicamentosas, considerando las preferencias y preocupaciones del paciente.

terapia familiar sistémica para lograr remisión de síntomas, mejor la calidad de vida, recuperación funcional, disminución del riesgo suicida, intento de suicidio y suicidios?

\section{De la evidencia científica a la práctica clínica}

No se encontraron artículos que comparen ambas intervenciones, pero se logró recuperar un artículo que reportaba la efi- cacia de la Terapia Familiar Sistémica (TFS). Una revisión sistemática de seis estudios observacionales reportó que cuando se compara la efectividad de la terapia familiar sistemática con la efectividad de intervenciones grupales, intervenciones cognitivas o de comportamiento individual, no queda claro si la terapia familiar sistémica es una intervención efectiva. No hay evidencia sobre las consecuencias potencialmente adversas de este tipo de intervención ${ }^{25}$. La calidad de la evidencia fue muy baja ya que tres de los seis estudios presentaron riesgo de sesgo.

\section{Beneficios clínicos y daños potencia-} les de esta recomendación

Ante la falta de evidencia para terapia de familia, los grupos de ayuda mutua podrían resultar potencialmente beneficiosos como una intervención en pacientes adultos. Los pacientes ven positivamente las intervenciones grupales y 
familiares como parte de un tratamiento integral de la depresión.

Pregunta 6. ¿Cuánto es el tiempo adecuado de tratamiento con psicoterapia (o número de sesiones necesarias) para obtener respuesta terapéutica, remisión de síntomas y recuperación funcional en pacientes con diagnóstico de depresión?

\section{De la evidencia científica a la práctica clínica}

Un estudio observacional encontró diferencias grupales significativas en el tiempo de remisión de síntomas en el grupo que recibió terapia combinada, en comparación con los grupos que recibieron solo farmacoterapia o solo psicoterapia. El tratamiento combinado de antidepresivo y psicoterapia resulta en remisiones más rápidas del cuadro depresivo a partir de la semana seis ${ }^{26}$. La calidad de evidencia fue moderada.

Un ensayo clínico no reportó diferencias estadísticamente significativas en las mediciones de resultados clínicos entre los grupos de tratamiento; es decir, que ambos grupos se beneficiaron de los tratamientos. No se encontraron diferencias significativas en cambios clínicos, entre los grupos, después de 12 meses de seguimiento ${ }^{27}$.

Otro estudio se realizó con el objetivo de determinar el número de sesiones psicoterapéuticas necesarias para la remisión de síntomas, utilizando CBASP (sistema de análisis cognitivo conductual de psicoterapia), dos veces por semana durante las semanas 1 - 4 y semanalmente durante las semanas 5-12 más un medicamento antidepresivo (nefazodone). Según sus resultados, a pesar que la reducción de síntomas antes del punto medio de tratamiento, no predijo significativamente el estado de remisión; la no remisión fue detectada tan temprano como el punto medio de tratamiento (semana seis). La predicción de punto medio fue particularmente buena entre aquellos que recibieron psicoterapia combinada con medicación antidepresiva ${ }^{28}$.

Beneficios clínicos y daños potenciales de esta recomendación

Los beneficios son considerados como moderados y los daños como pequeños, lo cual nos lleva a considerar que probablemente favorece a la intervención.

\section{EVENTOS ADVERSOS DE LA FARMACOTERAPIA}

Pregunta 7. En pacientes adultos con depresión leve o moderada, ¿EI uso de farmacoterapia es seguro en términos de interacciones medicamentosas y reacciones adversas?

\section{clínica}

Un metaanálisis en red de 117 ensayos clínicos comparó los siguientes antidepresivos en rango de dosis terapéutica para el tratamiento agudo de depresión: bupropion, citalopram, duloxetina, escitalopram, fluoxetina, fluvoxamina, milnacipran, mirtazapina, paroxetina, reboxetina, sertralina, y venlafaxine, para determinar tasa de respuesta y deserción. Al comparar escitalopram con mirtazapina, se encontró que mirtazapina presentó mayor eficacia, pero escitalopram presentó menos efectos adversos. Al comparar mirtazapina con sertralina se encontró que mirtazapina presentó mayor eficacia y sertralina tuvo menos eventos adversos. Los autores encontraron diferencias clínicas importantes entre los antidepresivos prescritos comúnmente tanto para eficacia como aceptabilidad a favor de escitalopram y sertralina ${ }^{29}$.

Una revisión sistematica con metaanálisis encontró que adicionar medicamentos antagonistas selectivos de serotonina y noradrenalina (NaSSA) a inhibidores selectivos de la recaptación de serotonina (IRSS) estuvo asociado específicamente con más de un evento adverso como sedación y ganancia de peso mayor o igual a $7 \%$, por lo que sugirieron tener precaución al utilizar este co-tratamiento ${ }^{30}$.

Beneficios clínicos y daños potenciales de esta recomendación

Los antidepresivos han demostrados ser eficaces en mejorar los síntomas depresivos. Cada grupo de antidepresivos tiene un perfil particular de eventos adversos. Por ello, el médico debe decidir junto con el paciente cual es el antidepresivo que más le conviene. Si el paciente es informado por su médico de los efec- tos adversos, los tolerará mejor. Los daños potenciales se relacionan al abandono el tratamiento por causa de eventos adversos indeseables o poco tolerables para el paciente. Los eventos adversos que afectan a la esfera sexual son los menos tolerados tanto por varones como por mujeres.

\section{RECOMENDACIONES PARA LA INVESTIGACIÓN}

Durante el dialogo deliberativo para la formulación de recomendaciones aplicando la metodología GRADE se identificaron necesidades de investigación, que pueden ser incluidas dentro de las prioridades de investigación en salud mental en el país y de esta modo se puede contribuir con el manejo de la depresión en adultos en el contexto peruano; estas son: la evaluación de la efectividad entre distintas psicoterapias individuales en el seguimiento de los pacientes tratados y la evaluación de la efectividad de las psicoterapias grupales en el tratamiento de la depresión.

\section{AGRADECIMIENTOS}

A la Dra. Elizabeth Rivera Chávez directora del Hospital Víctor Larco Herrera (HVLH) por impulsar la realización de la guía. Al Dr. Ricardo Bustamante Quiroz médico psiquiatra del HVLH y al Dr. Ludovic Reveiz Herault médico epidemiólogo de la Organización Panamericana de la Salud sede Washington, por la revisión clínica y metodológica respectivamente. Al equipo de la oficina de gestión de calidad del HVLH por impulsar y acompañar todo el proceso.

\section{REFERENCIAS BIBLIOGRÁFICAS}

1. Organización Mundial de la Salud [Internet]. Depresión, Nota descriptiva 2017 [Fecha de acceso: 15 de enero 2019]. Disponible en: http://www.who.int/ mediacentre/factsheets/fs369/es/

2. Ministerio de Salud. Plan Nacional de fortalecimiento de servicios de salud mental comunitaria 20182021. 2018

3. Organización Mundial de la Salud [Internet]. Depresión. Temas de Salud 2017 [Fecha de acceso: 15 de enero 2019]. Disponible en: http://www.who.int/ topics/depression/es/

4. Ministerio de Salud del Perú [Internet]. Dirección General de Salud de las Personas, Dirección de 
Salud Mental. Guia técnica: Guía de Práctica Clínica en Depresión, RM Nº48-2006 [Fecha de acceso: 15 de enero 2019]. Disponible en: http://bvs.minsa. gob.pe/local/MINSA/1084_DGSP261.pdf

5. Ministerio de Salud del Perú [Internet]. NTS N¹38MINSA/2017/DGIESP: Norma técnica de Salud de Centros de Salud Mental Comunitarios. RM N ${ }^{\circ} 574-$ 2017/MINSA [Fecha de acceso: 15 de enero 2019]. Disponible en: https://cdn.www.gob.pe/uploads/ document/file/189591/189078_RM_N_C2_B05742017-MINSA.pdf20180823-24725-1pfq378.pdf

6. Ministerio de Salud del Perú [Internet]. Directiva Administrativa que establece la cartera de atención de salud en los establecimientos de salud del primer nivel de atención. DS N²42-MINSA/2017 DGAIN [Fecha de acceso: 15 de enero 2019]. Disponible en: https://cdn.www.gob.pe/uploads/ document/file/188529/188027_RM_N_C2_B0_1069 2017-MINSA_1.PDF20180823-24725-1mlafbs.PDF

7. Hospital Victor Larco Herrera [Internet]. Guía de práctica clínica para el tratamiento de depresión en adultos-versión extensa. RD N¹32-2018-DG-HVLH [Fecha de acceso: 15 de enero 2019]. Disponible en: https://www.larcoherrera.gob.pe/images/ documentos/resoluciones/2018/RD_132_2018_ DG_HVLH.pdf

8. Ministerio de Salud del Perú [Internet]. Dirección General de Salud de las Personas, Dirección de Calidad en Salud. Documento Técnico: Metodología para la elaboración de guias de práctica clínica, RM N414-2015/MINSA [Fecha de acceso: 15 de enero 2019]. Disponible en: http://bvs.minsa.gob. pe/local/MINSA/3301.pdf

9. Organización Mundial de la Salud. Guía de bolsillo de la clasificación CIE-10. Clasificación de los trastornos mentales y del comportamiento. Madrid, España: Médica Panamericana; 1994.

10. Organización Panamericana de la Salud [Internet] OPS, OMS, Mental Health Gap Action Programme. Guia de intervención mhGAP para los trastornos mentales, neurológicos y por consumo de sustancias en el nivel de atención de salud no especializada. Versión 2.0. 2017. [Fecha de acceso: 15 de enero 2019]. Disponible en: http://iris.paho.org/ xmlui/handle/123456789/34071

11. Ministerio de Salud del Perú [Internet]. Documento Técnico Metodologia para la elaboración de Guias de Práctica Clínica [Fecha de acceso: 15 de enero 2019]. 2015. Disponible en: http://www.minsa.gob. pe/dgsp/documentos/Guias/M-GPC1.pdf

12. Consorcio AGREE [Internet]. Instrumento para la evaluación de Guías de Práctica Clínica, AGREE II, 2009 [Fecha de acceso: 15 de enero 2019]. Disponible en: http://www.guiasalud.es/contenidos/ documentos/Guias_Practica_Clinica/SpanishAGREE-II.pdf
13. Ministerio de Salud y Protección Social de Colombia [Internet]. Ministerio de Salud y Protección Social de Colombia, Instituto de Evaluación Tecnológica en Salud, Departamento Administrativo de Ciencia Tecnologia e Innovación-COLCIENCIAS. Guía de Práctica Clínica (GPC) Detección temprana y diagnóstico del episodio depresivo y trastorno depresivo recurrente en adultos. Atención integral de los adultos con diagnóstico de episodio depresivo o trastorno depresivo recurrente 2013 [Fecha de acceso: 15 de enero 2019]. Disponible en: http://gpc. minsalud.gov.co/gpc_sites/Repositorio/Conv_500/ GPC_td/GPC_td_completa.aspx

14. Ministerio de Sanidad España [Internet]. Ministerio de Sanidad de España, Servicios Sociales e Igualdad, Agencia de Evaluación de Tecnologias Sanitarias de Galicia. Guia de Práctica Clínica sobre el Manejo de la Depresión en el Adulto 2014 [Fecha de acceso: 15 de enero 2019]. Disponible en: http:// www.guiasalud.es/GPC/GPC_534_Depresion_Adulto_Avaliat_compl.pdf

15. Grupo de trabajo GRADE [Internet]. Manual GRADE (Grading of Recommendations, Assessment, Development and Evaluation), Versión en Español 2017 [Fecha de acceso: 15 de enero 2019]. Disponible en: http://gdt.guidelinedevelopment.org/app/

16. McMaster University [Internet]. Mac GRADE, Centre McMaster University. GIN-McMaster Lista de comprobación del proceso de elaboración de guias de práctica clínica 2014 [Fecha de acceso: 15 de enero 2019]. Disponible en: https://cebgrade.mcmaster. ca/GDCtranslations/guidelinechecklistspanish.ht ml\#GuidelineGroupMembershiptable

17. Carmona G, Bonilla C, Huamán K, Reyes N, Hijar G, Caballero P. Utilidad de los diálogos deliberativos para la formulación de recomendaciones de guias de práctica clínica. Rev. Perú. Med Exp Salud Pública. 2017;3(4):738-43. DOI: http://dx.doi. org/10.17843/rpmesp.2017.344.3347

18. Fournier JC, DeRubeis RJ, Hollon SD, Dimidjian S, Amsterdam JD, Shelton RC, et al. Antidepressant drug effects and depression severity: a patient-level meta-analysis. JAMA. 2010;303(1):47-53. DOI: 10.1001/jama.2009.1943

19. Driessen E, Cuijpers P, Hollon SD, Dekker JJM. Does pretreatment severity moderate the efficacy of psychological treatment of adult outpatient depression? A meta-analysis. J Consult Clin Psychol. 2010;78(5):668-80. DOI: 10.1037/a0020570

20. Furukawa TA, Weitz ES, Tanaka S, Hollon SD, Hofmann SG, Andersson G, et al. Initial severity of depression and efficacy of cognitive-behavioural therapy: individual-participant data meta-analysis of pill-placebo-controlled trials. Br J Psychiatry J Ment Sci. 2017; 210(3):190-6. DOI: 10.1192/bjp. bp. 116.187773
21. Cuijpers P, Dekker J, Hollon SD, Andersson G. Adding psychotherapy to pharmacotherapy in the treatment of depressive disorders in adults: a meta-analysis. $J$ Clin Psychiatry. 2009;70(9):1219-29. DOI: 10.4088/ JCP.09r05021

22. Ministerio de Salud del Perú [Internet]. Ministerio de Salud del Perú. Dirección General de Intervenciones Estratégicas en Salud Pública, Direcció de Salud Mental. Boletín de gestión: Salud Mental Comunitaria Nuevo modelo de atención 2016 [Fecha de acceso: 15 de enero 2019]. Disponible en: http://bvs.minsa.gob. pe/local/MINSA/3615.pdf

23. Cuijpers P, Berking M, Andersson G, Quigley L, Kleiboer A, Dobson KS. A meta-analysis of cognitivebehavioural therapy for adult depression, alone and in comparison with other treatments. Can J Psychiatry Rev Can Psychiatr. 2013;58(7):376-85. DOI: 10.1177/070674371305800702

24. David D, Szentagotai A, Lupu V, Cosman D. Rationa emotive behavior therapy, cognitive therapy, and medication in the treatment of major depressive disorder: a randomized clinical trial, posttreatment outcomes, and six-month follow-up. J Clin Psychol. 2008;64(6):728-46. DOI: $10.1002 /$ jclp.20487

25. Henken HT, Huibers MJH, Churchill R, Restifo K, Roelofs J. Family therapy for depression. Cochrane Database Syst Rev. 2007;(3):CD006728. DOI: 10.1002/14651858. CD006728

26. Manber R, Kraemer HC, Arnow BA, Trivedi MH, Rush AJ, Thase ME, et al. Faster remission of chronic depression with combined psychotherapy and medication than with each therapy alone. J Consult Clin Psychol. 2008;76(3):459-67. DOI: 10.1037/0022-006X.76.3.459

27. Kontunen J, Timonen M, Muotka J, Liukkonen T. Is interpersonal counselling (IPC) sufficient treatment for depression in primary care patients? A pilot study comparing IPC and interpersonal psychotherapy (IPT). J Affect Disord. 2016;189:89-93. DOI: 10.1016/J. jad.2015.09.032

28. Steidtmann D, Manber R, Blasey C, Markowitz JC, Klein DN, Rothbaum BO, et al. Detecting critical decision points in psychotherapy and psychotherapy + medication for chronic depression. J Consult Clin Psychol. 2013;81(5):783-92. DOI: 10.1037/a0033250

29. Cipriani A, Furukawa TA, Salanti G, Geddes JR, Higgins JP, Churchill $R$, et al. Comparative efficacy and acceptability of 12 new-generation antidepressants: a multiple-treatments meta-analysis. Lancet Lond Engl. 2009;373(9665):746-58. DOI: 10.1016/S01406736(09)60046-5

30. Galling B, Calsina Ferrer A, Abi Zeid Daou M, Sangroula D, Hagi K, Correll CU. Safety and tolerability of antidepressant co-treatment in acute major depressive disorder: results from a systematic review and exploratory meta-analysis. Expert Opin Drug Saf. 2015;14(10):1587608. DOI: 10.1517/14740338.2015.1085970 